Unfolding the fission prompt gamma-ray energy and multiplicity distribution measured by DANCE

A. Chyzh, C. Y. Wu, T. Bredeweg, A. Couture, M. Jandel, J. Ullmann, A. Laptev

October 19, 2010 
This document was prepared as an account of work sponsored by an agency of the United States government. Neither the United States government nor Lawrence Livermore National Security, LLC, nor any of their employees makes any warranty, expressed or implied, or assumes any legal liability or responsibility for the accuracy, completeness, or usefulness of any information, apparatus, product, or process disclosed, or represents that its use would not infringe privately owned rights. Reference herein to any specific commercial product, process, or service by trade name, trademark, manufacturer, or otherwise does not necessarily constitute or imply its endorsement, recommendation, or favoring by the United States government or Lawrence Livermore National Security, LLC. The views and opinions of authors expressed herein do not necessarily state or reflect those of the United States government or Lawrence Livermore National Security, LLC, and shall not be used for advertising or product endorsement purposes.

This work performed under the auspices of the U.S. Department of Energy by Lawrence Livermore National Laboratory under Contract DE-AC52-07NA27344. 
LLNL-TR-460216

\title{
Unfolding the fission prompt $\gamma$-ray energy and multiplicity distribution measured by DANCE
}

\author{
A. Chyzh and C.Y. Wu \\ Lawrence Livermore National Laboratory, Livermore, CA 94551 \\ T. Bredeweg, A. Couture, M. Jandel, J. Ullmann, and A. Laptev \\ Los Alamos National Laboratory, Los Alamos, NM 87545
}

(Dated: October 19, 2010)

\begin{abstract}
The nearly energy independence of the $\gamma$-ray efficiency and multiplicity response for the DANCE array, the unusual characteristic elucidated in our early technical report (LLNL-TR-452298), gives one a unique opportunity to derive the true prompt $\gamma$-ray energy and multiplicity distribution in fission from the measurement. This unfolding procedure for the experimental data will be described in details and examples will be given to demonstrate the feasibility of reconstruction of the true distribution.
\end{abstract}

Keywords: DANCE, GEANT4, fission gamma-ray energy, multiplicity. 


\section{INTRODUCTION}

DANCE (Detector for Advanced Neutron Capture Experiments [1]) is a $\gamma$-ray calorimeter and designed to study the neutron-capture reactions on small quantities of radioactive and rare stable nuclei. These reactions are important for the radiochemistry applications and modeling the element production in stars. The recognition of the capture event is made by the summed $\gamma$-ray energy $\mathrm{E}_{\sum}$ which is equivalent of the reaction $\mathrm{Q}$-value and unique for a given capture reaction. For a selective group of actinide nuclei, where the fission reaction competes favorably with the capture reaction, additional signature is needed to distinguish between fission and capture $\gamma$-rays for the DANCE measurement. This can be accomplished by introducing a detector system to detect fission fragments, which establishes a unique signature for the fission event. Once this system is implemented, one has the opportunity to study not only the capture but also fission reaction.

For the fission reaction, one has a unique opportunity to study the prompt $\gamma$-ray energy and multiplicity distribution by taking advantage of the unusual characteristic of DANCE, which was reported in our earlier technical writing (LLNL-TR-452298). Here we describe an unfolding procedure to reconstruct the true $\gamma$-ray energy and multiplicity distribution in fission from the DANCE measurement. The validity of this unfolding procedure is established by examining the cases for three radioactive sources, ${ }^{60} \mathrm{Co},{ }^{88} \mathrm{Y}$, and ${ }^{22} \mathrm{Na}$. The plan to measure these quantities for a selective group of actinide nuclei will be discussed.

\section{$\gamma$-RAY MULTIPLICITY}

In the DANCE data analysis we utilize a concept of cluster multiplicity $\mathrm{M}_{c l}$, where a cluster is a group of adjacent crystals that fire above the $150-\mathrm{keV}$ energy threshold each within a given time coincidence window, typically $\mathrm{T}_{\text {coin }}=5-20$ ns. Because of the finite efficiency, the number of $\gamma$ rays detected by DANCE is not always equal to the number of emitted $\gamma$-rays, the true $\gamma$-ray multiplicity $\mathrm{M}_{\gamma}$.

Fig. 1 shows the simulated $\mathrm{M}_{c l}$ response of DANCE to the emitted (true) $\mathrm{M}_{\gamma}$. From now on the words emitted and true are interchangable. Each plot contains three spectra and each spectrum was simulated with $10^{5}$ mono energetic $\gamma$-rays: $\mathbf{0 . 5} \mathbf{M e V}$ in black, $1 \mathrm{MeV}$ in red, $2 \mathrm{MeV}$ in blue. For the event with the true $\gamma$-ray multiplicity $\mathrm{M}_{\gamma}=1$, the total efficiency 

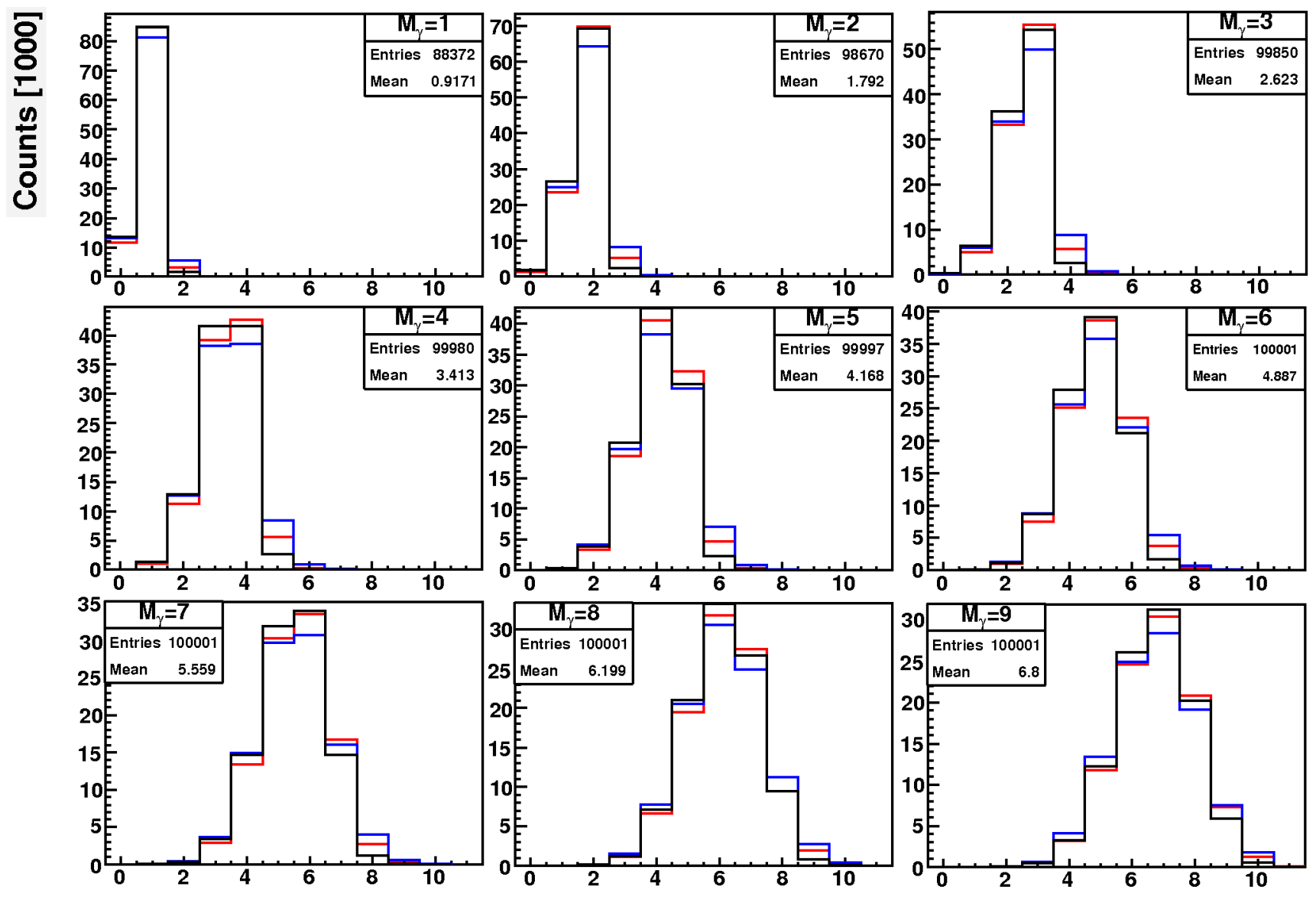

Cluster Multiplicity

FIG. 1: The $\mathrm{M}_{c l}$ spectra for different $\mathrm{E}_{\gamma} .<\mathrm{M}>$ mean value is a function of $\mathrm{M}_{c l}$. The spectrum integrals (Entries) do not include the $\mathrm{M}_{c l}=0$ counts.

$\epsilon_{\text {tot }}(0.5 \mathrm{MeV})$ is $88.3 \%$, from which $\epsilon(M=1)=85 \%, \epsilon(M=2)=2 \%$, and $\epsilon(M=0)=13 \%$. Thus, the measured mean $\left\langle\mathrm{M}_{c l}>=0.92\right.$ corresponds to the real $\mathrm{M}_{\gamma}=1$. The dependece of the mean value $\left\langle\mathrm{M}_{c l}>\right.$ from $\mathrm{M}_{\gamma}$ for 3 different $\gamma$-ray energies is shown in Tab I and Fig. 2.

The DANCE response to the $\mathrm{M}_{\gamma}=2$ events spans over the cluster multiplicity between 1 and 4 ; the $\mathrm{M}_{\gamma}=6$ events are detected anywhere between $\mathrm{M}_{c l}=2$ and 8 with corresponding probabilities shown in Fig. 1. Thus, what we measure with DANCE is not the true $\gamma$-ray multiplicity $\mathrm{M}_{\gamma}$, but rather the $\mathrm{M}_{c l}$ distribution folded through the DANCE response. 
TABLE I: Mean values $\left\langle\mathrm{M}_{c l}>\right.$ of measured $\mathrm{M}_{c l}$ as a function of true $\mathrm{M}_{\gamma}$ for 3 different energies $\mathrm{E}_{\gamma}$. Each row/column corresponds to a certain $\mathrm{M}_{c l} / \mathrm{E}_{\gamma}$.

\begin{tabular}{|l|c|c|c|}
\hline & $\mathrm{E}_{\gamma}=0.5 \mathrm{MeV}$ & $\mathrm{E}_{\gamma}=1 \mathrm{MeV}$ & $\mathrm{E}_{\gamma}=2 \mathrm{MeV}$ \\
\hline $\mathrm{M}_{\gamma}=1$ & 1.02 & 1.04 & 1.06 \\
\hline $\mathrm{M}_{\gamma}=2$ & 1.75 & 1.81 & 1.84 \\
\hline $\mathrm{M}_{\gamma}=3$ & 2.53 & 2.62 & 2.64 \\
\hline $\mathrm{M}_{\gamma}=4$ & 3.31 & 3.41 & 3.42 \\
\hline $\mathrm{M}_{\gamma}=5$ & 4.05 & 4.16 & 4.17 \\
\hline $\mathrm{M}_{\gamma}=6$ & 4.75 & 4.89 & 4.88 \\
\hline $\mathrm{M}_{\gamma}=7$ & 5.44 & 5.55 & 5.54 \\
\hline $\mathrm{M}_{\gamma}=8$ & 6.09 & 6.20 & 6.16 \\
\hline $\mathrm{M}_{\gamma}=9$ & 6.71 & 6.80 & 6.73 \\
\hline
\end{tabular}

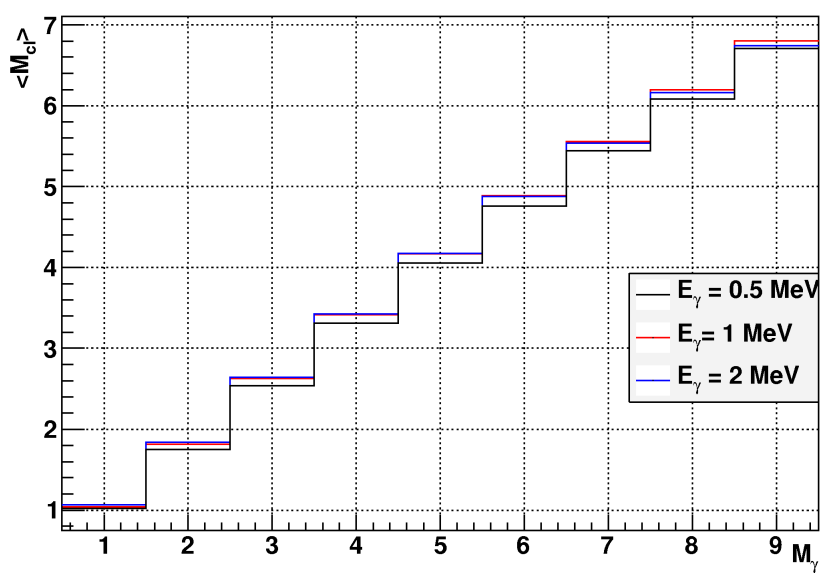

FIG. 2: Mean values of $\mathrm{M}_{c l}$ depend on true $\mathrm{M}_{\gamma}$ and almost don not depend on $\mathrm{E}_{\gamma}$.

\section{M-response matrix of DANCE}

The transition from $\mathrm{M}_{\gamma}$ to $\mathrm{M}_{c l}$ is determined by the DANCE response and can be arranged into the M-response matrix which explicitly describes dependencies between these two quantities.

Fig. 3 shows how the M-response matrix is structured. The $\mathrm{X}$ axis corresponds to the true multiplicity $\mathrm{M}_{\gamma}$, and the $\mathrm{Y}$ axis corresponds to the measured multiplicity $\mathrm{M}_{c l}$. For instance, 100,000 events of $\mathrm{M}_{\gamma}=1$ event are measured as follows: 65,100 events are $\mathrm{M}_{c l}=1$, 3,300 events are $\mathrm{M}_{c l}=2$, and 11 events are $\mathrm{M}_{c l}=3$. Another example is how the $\mathrm{M}_{\gamma}=2$ events are measured: $23.5 \%$ are measured as $\mathrm{M}_{c l}=1,69.8 \%$ are $\mathrm{M}_{c l}=2,5.2 \%$ are $\mathrm{M}_{c l}=3$, and so forth (Tab. II). The $\mathrm{M}_{c l}=0$ values represent events that are not detected by DANCE. 


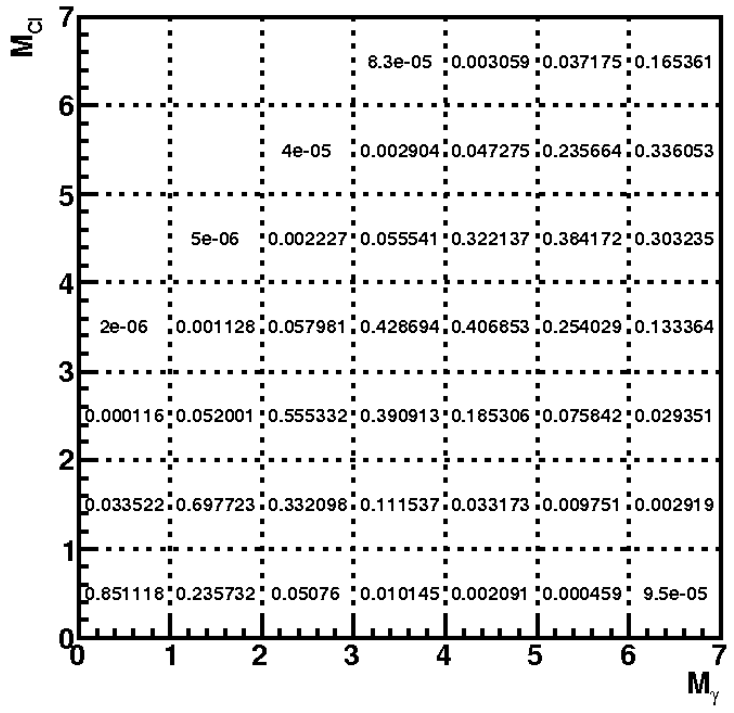

FIG. 3: M-response matrix of DANCE. Each columns corresponds to a $\mathrm{M}_{\gamma}$ value and contains $\mathrm{M}_{c l}$ values.

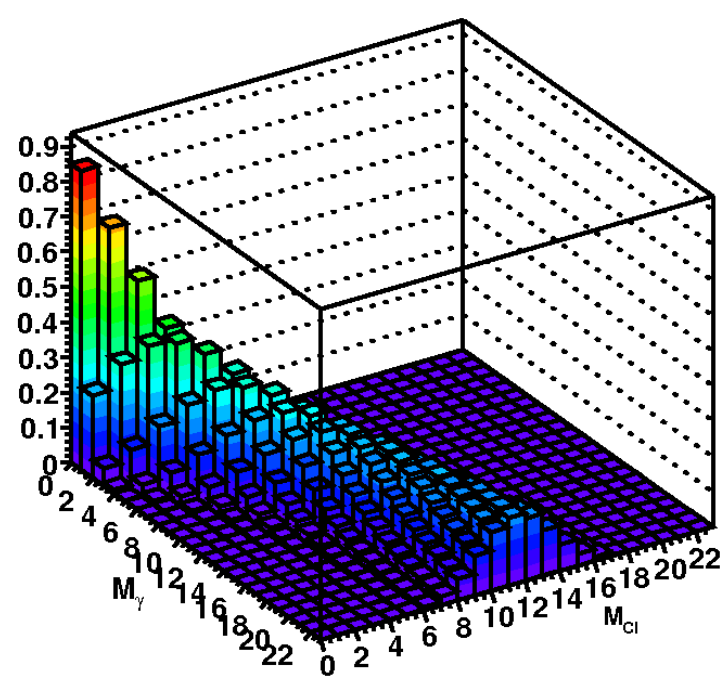

FIG. 4: M-response matrix of DANCE. 3D view.

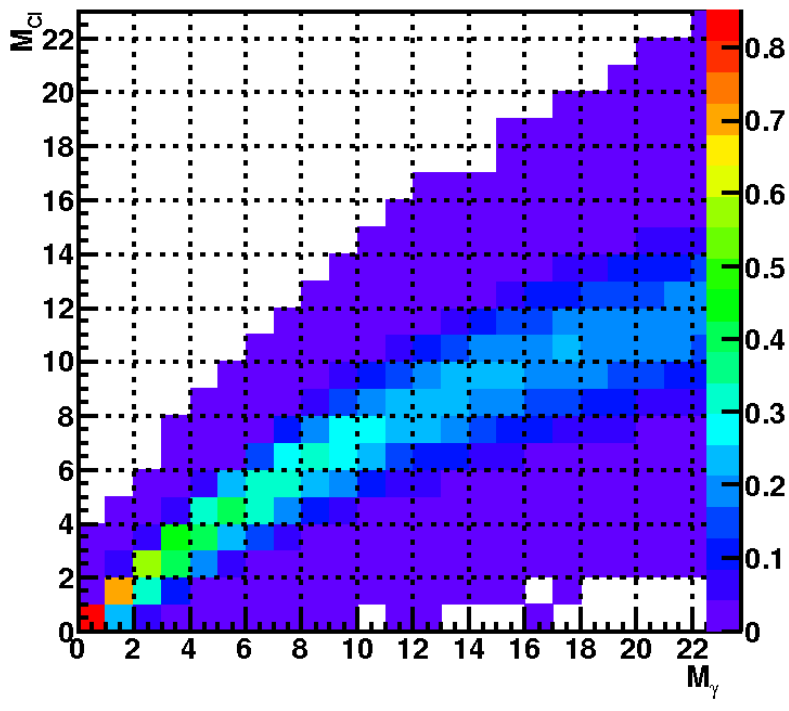

FIG. 5: M-response matrix of DANCE. The multiplicity range is $\mathrm{M}=1-23$.

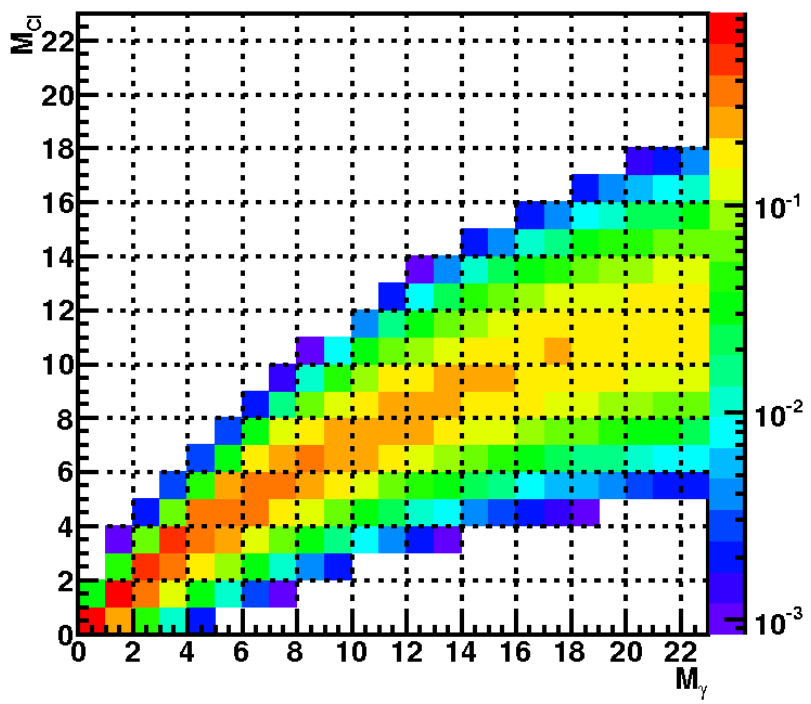

FIG. 6: M-response matrix of DANCE. Z-axis log view.

TABLE II: DANCE response to true $\mathrm{M}_{\gamma}$ is distributed over $\mathrm{M}_{c l}$ values

\begin{tabular}{|l|l|l|l|}
\hline & $\mathrm{M}_{\gamma}=1$ & $\mathrm{M}_{\gamma}=2$ & $\mathrm{M}_{\gamma}=3$ \\
\hline $\mathrm{M}_{c l}=0$ & $11.7 \%$ & $1.3 \%$ & $0.1 \%$ \\
\hline $\mathrm{M}_{c l}=1$ & $85.0 \%$ & $23.5 \%$ & $5.0 \%$ \\
\hline $\mathrm{M}_{c l}=2$ & $3.3 \%$ & $69.8 \%$ & $33.2 \%$ \\
\hline $\mathrm{M}_{c l}=3$ & $0.0 \%$ & $5.2 \%$ & $55.5 \%$ \\
\hline $\mathrm{M}_{c l}=4$ & $0.0 \%$ & $0.01 \%$ & $5.7 \%$ \\
\hline $\mathrm{M}_{c l}=5$ & $0.0 \%$ & $0.0 \%$ & $0.2 \%$ \\
\hline
\end{tabular}


The colored representations of the M-response matrix (Fig. 5 and 6) show that the multiplicity range spans up to $\mathrm{M}=23$. The number of $\mathrm{BaF}_{2}$ crystals in DANCE is 160 , but the multiplicity cannot go that high for two reasons: a cluster may contain several crystals, and clusters may overlap thus reducing $\mathrm{M}_{c l}$ value. That's why the matrix is truncated to the size of 23 for the practical purpose. The columns of the matrix become increasingly linearly dependent on each other, i.e., as $\mathrm{M}_{\gamma}$ increases, the dependence of $\mathrm{M}_{c l}$ on $\mathrm{M}_{\gamma}$ decreases. The more linearly dependent matrix is, the more difficult is to solve it. That is, solving the matrix becomes nearly impossible once its determinant closes to zero within the limits of computer accuracy. Fig. 4 is a 3D view of the M-response matrix.

It is necessary to mention that the present matrix is built for the $1-\mathrm{MeV}$ mono energetic photons. The same matrix for the $2-\mathrm{MeV}$ photons would look slightly different because the DANCE detection efficiency weakly depends on the $\gamma$-ray energy $\mathrm{E}_{\gamma}$ (see Ref. [3]), where the probabilities for detecting the $\mathrm{M}=1$ events with the $1 \mathrm{MeV}$ and $2 \mathrm{MeV}$ energies are $87 \%$ and $85 \%$ correspondingly. For the higher multiplicity case such as $\mathrm{M}_{\gamma}=5$, the largest $\mathrm{M}_{c l}$ values varies less than $5 \%$, but the total area of the $\mathrm{M}_{c l}$ distribution, which is the total efficiency, changes only less than $2 \%$. The majority of $\gamma$-rays produced in the neutron capture and neutron induced fission reactions on many nuclei lay within $100 \mathrm{keV}$ to several $\mathrm{MeV}$. Thus, for the first approximation, this M-response matrix can be used to obtain true $\mathrm{M}_{\gamma}$ distributions without knowing the exact $\gamma$-ray energy if we assume the M-response matrix for $1 \mathrm{MeV}$ is the same for other energies.

\section{Multiplicity unfolding}

The M-response matrix can be used to derive the true $\gamma$-ray multiplicity $\mathrm{M}_{\gamma}$ from the measured cluster multiplicity $\mathrm{M}_{c l}$, the so-called unfolding procedure. Also the same matrix can be used for folding the true $\mathrm{M}_{\gamma}$ into the measured $\mathrm{M}_{c l}$.

The folding procedure can be demonstrated in Fig 7. The Brunson distribution [4] for the ${ }^{252} \mathrm{Cf}$ fission prompt $\gamma$-ray output (red) represents a true $\mathrm{M}_{\gamma}$ distribution. The $\mathrm{M}_{c l}$ distribution (black) is the simulated DANCE response to the Brunson distribution, i.e., the Brunson distribution is fed into the GEANT4 model of DANCE as an input and the $\mathrm{M}_{c l}$ distribution is the output. The folded $\mathrm{M}_{\text {fold }}$ distribution (blue) is the matrix product. The 
folding procedure is the matrix product:

$$
V\left(M_{c l}\right)=R(M) \cdot V\left(M_{\gamma}\right)
$$

where $V\left(M_{c l}\right)$ is the vector of the $\mathrm{M}_{c l}$ values, $R(M)$ is the M-response matrix, and $V\left(M_{\gamma}\right)$ is the vector of the $\mathrm{M}_{\gamma}$ values. Before taking the matrix product of $R(M)$ and $V\left(M_{\gamma}\right)$ both of them need to be truncated to the same dimensions. The folding procedure should generate $\mathrm{M}_{\text {fold }}$ distribution equal to the $\mathrm{M}_{c l}$ distribution. The agreement between these two is very good but not perfect. For $\mathrm{M}=16$ and higher the folded $\mathrm{M}$ distribution remains constant instead of decreasing as it should. The computer accuracy is limited by 64 bits (type double in $\mathrm{C}++$ and Double_t in ROOT).

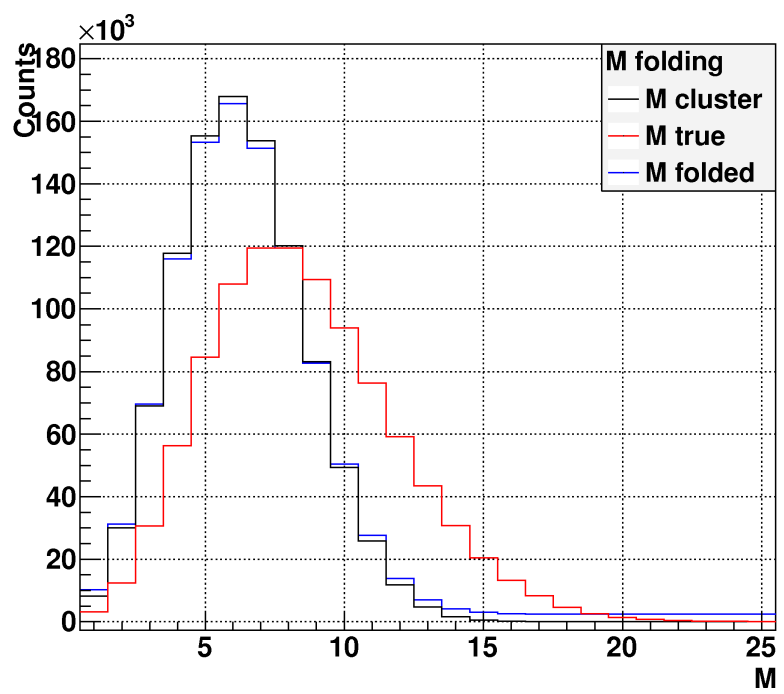

FIG. 7: Folding Brunson distribution of $\mathrm{M}_{\gamma}$ into cluster multiplicity $\mathrm{M}_{c l}$.

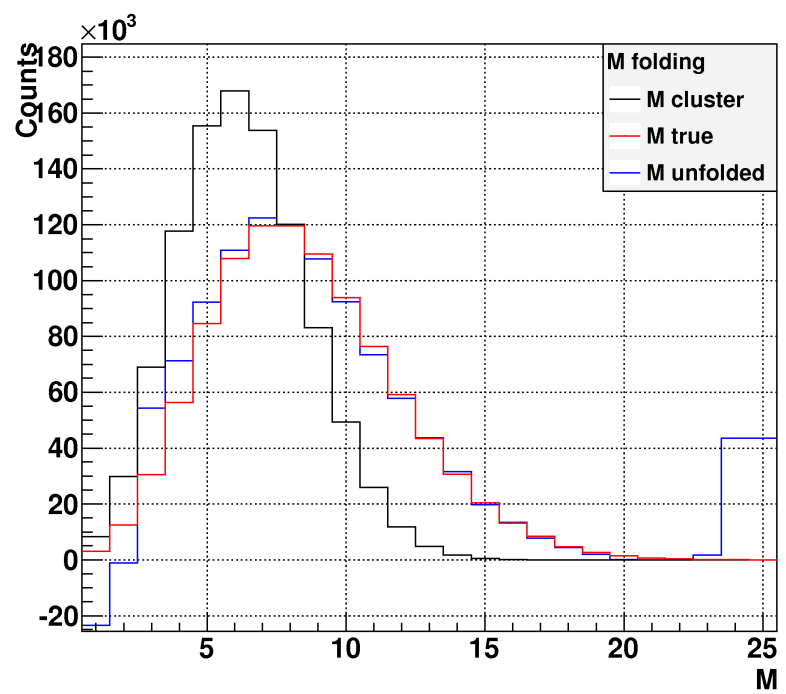

FIG. 8: M-response matrix of DANCE. The multiplicity range is $\mathrm{M}=1-23$

The unfolding procedure is more complicated than folding. The most straight forward way to unfold the measured $\mathrm{M}_{c l}$ into the true $\mathrm{M}_{\gamma}$ is to invert the matrix $R(M)$ and take the matrix product:

$$
V\left(M_{\gamma}\right)=R^{-1}(M) \cdot V\left(M_{c l}\right)
$$

where $R^{-1}(M)$ is the inversion of the M-response matrix $R(M)$. The inversion of the $23 \times 23$ matrix precisely is difficult if possible at all because of the increasing linear dependence of matrix elements. If the inversion is precise, product $R(M) \times R^{-1}(M)$ will produce a unit matrix $I$ with diagonal elements equal to 1 and off-diagonal equal to 0 . But in this case the 
$I$ matrix is not completely unit, some off-diagonal elements fluctuate in the range of $10^{-16}$ $-10^{-3}$. Solving $R(M)$ by taking its inversion produces unrealistic fluctuation in the $V\left(M_{\gamma}\right)$ values.

The other way to solve Eq. 1 relative to the $V\left(M_{c l}\right)$ vector is to decompose the $R(M)$ matrix using Singular Value Decomposition (SVD) method [5]. The SVD method is a factorization of a rectangular or square real matrix. if $R$ is an $n \times n$ matrix with real nonnegative numbers, then there exists a factorization of the form:

$$
R=U Z V^{*}
$$

where $\mathrm{U}$ is an $n \times n$ unit matrix, $\mathrm{Z}$ is an $n \times n$ diagonal matrix with nonnegative real numbers on the diagonal, and $V^{*}$ is the conjugate transpose of $V$. The SVD method orders the diagonal entries of $\mathrm{Z}$ to be in descending order, then $\mathrm{Z}$ is uniquely determined by $R$, although $U$ and $V$ may not be unique. The diagonal values of $\mathrm{Z}$ are singular values of $R$.

Using the SVD method Eq. 1 can be solved relative to $V\left(M_{c l}\right)$ with a unique solution stored in the main diagonal of the $\mathrm{Z}$ matrix. Fig 8 shows the unfolding procedure for the Brunson $\mathrm{M}_{\gamma}$ distribution. The $\mathrm{M}$ true is in red and the $\mathrm{M}$ unfolded is in blue. The solution has some deviations in the range $\mathrm{M}=1-5$, other than that the agreement is very good. No manual or automatic fitting was performed here yet, although it can be done to remove the deviations by iterations to achieve a better agreement. This figure shows that unfolding of the true $\gamma$-ray multiplicity $\mathrm{M}_{\gamma}$ from the measured cluster multiplicity $\mathrm{M}_{c l}$ is indeed possible, thus, DANCE can deliver a true $\mathrm{M}_{\gamma}$ distribution without knowing the true $\gamma$-ray energy.

\section{$\gamma$-RAY ENERGY}

DANCE is a $4 \pi \gamma$-ray calorimeter and it can measure the total energy of an event $\mathrm{E}_{\sum}$, as well as individual $\gamma$-ray energies $\mathrm{E}_{\gamma}$. The energy of one cluster $\mathrm{E}_{c l}$ corresponds to the $\gamma$-ray energy deposited in the crystals adjacent to each other. The relation between $\mathrm{E}_{c l}$ and $\mathrm{E}_{\gamma}$ is

shown in Fig. 9. Each measured (simulated) $\mathrm{E}_{c l}$ spectrum is the DANCE response to the single true $\mathrm{E}_{\gamma}$ energy. The studied range is $0.30-10 \mathrm{MeV}$, these spectra were simulated using the GEANT4 model of DANCE (see Ref. [2]) and it was validated in Ref. [3]. 

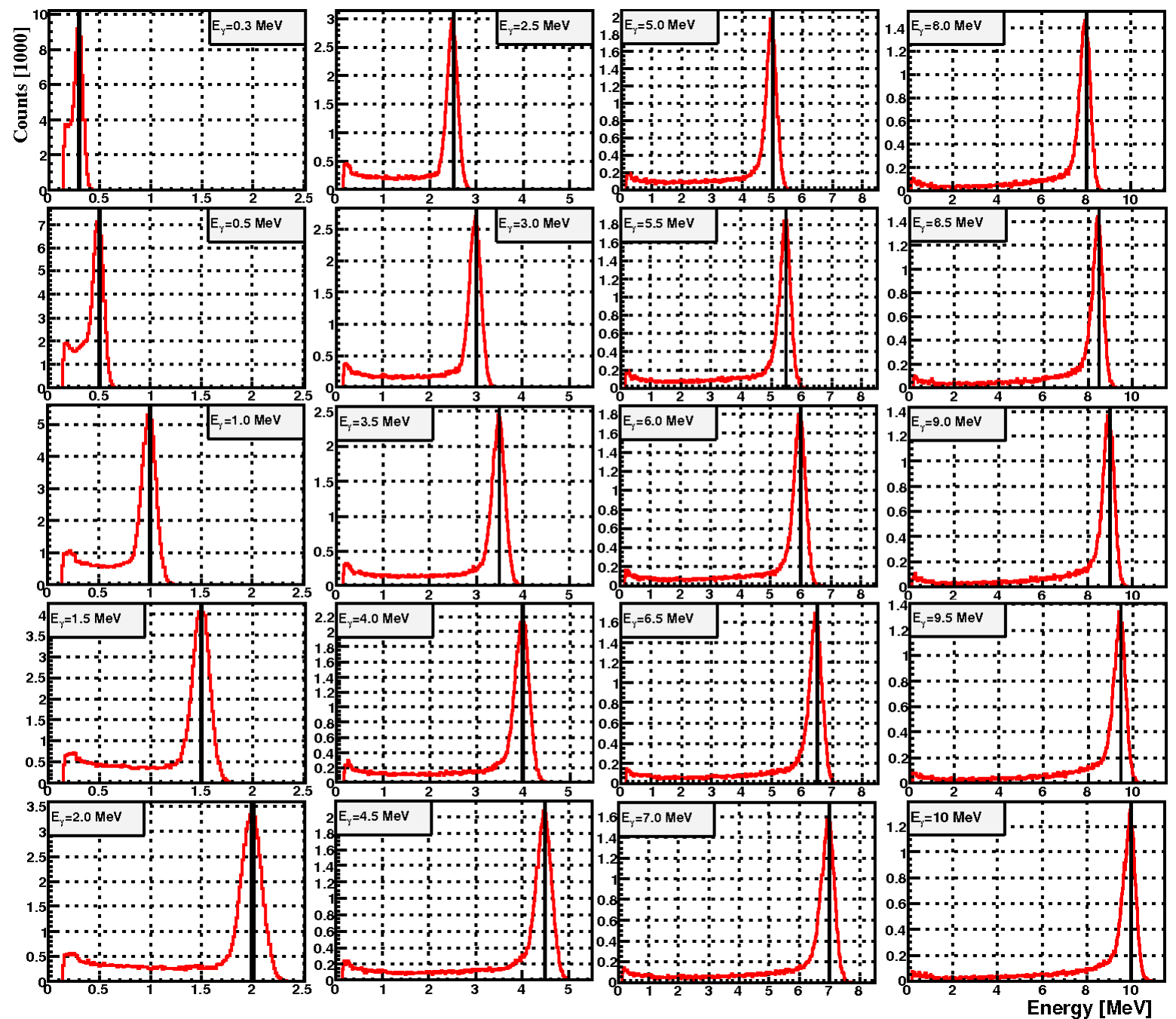

FIG. 9: Each $\mathrm{E}_{c l}$ energy corresponds to the true $\mathrm{E}_{\gamma}$.

\section{E-response matrix of DANCE}

The DANCE energy response to $1 \mathrm{MeV} \gamma$-ray is not just a photopeak at $1 \mathrm{MeV}$, but also a compton distribution from the $1-\mathrm{MeV}$ peak down to the $150-\mathrm{keV}$ threshold (see Fig. 9). Obviously, the measured energy $E_{c l}$ is a function of the true $\gamma$-ray energy $E_{\gamma}$, and for each $\mathrm{E}_{\gamma}$ value there has a unique $\mathrm{E}_{c l}$ response. Thus, the E-response matrix can be built for DANCE (see Fig. 10). For a given energy of $\mathrm{E}_{\gamma}$, the $\mathrm{E}_{c l}$ spectrum (DANCE response) is produced through simulations and normalized to the true number of $\gamma$-rays. Then it is integrated with the $50-\mathrm{keV}$ increment in the range $150 \mathrm{keV}-5 \mathrm{MeV}$.

The E-matrix is filled for $\mathrm{E}_{\gamma}=150 \mathrm{keV}$ to $5 \mathrm{MeV}$, total of 97 bins, each bin is $50 \mathrm{keV}$ wide. The lower limit of $150 \mathrm{keV}$ is the DANCE energy threshold and the upper limit of $5 \mathrm{MeV}$ is 


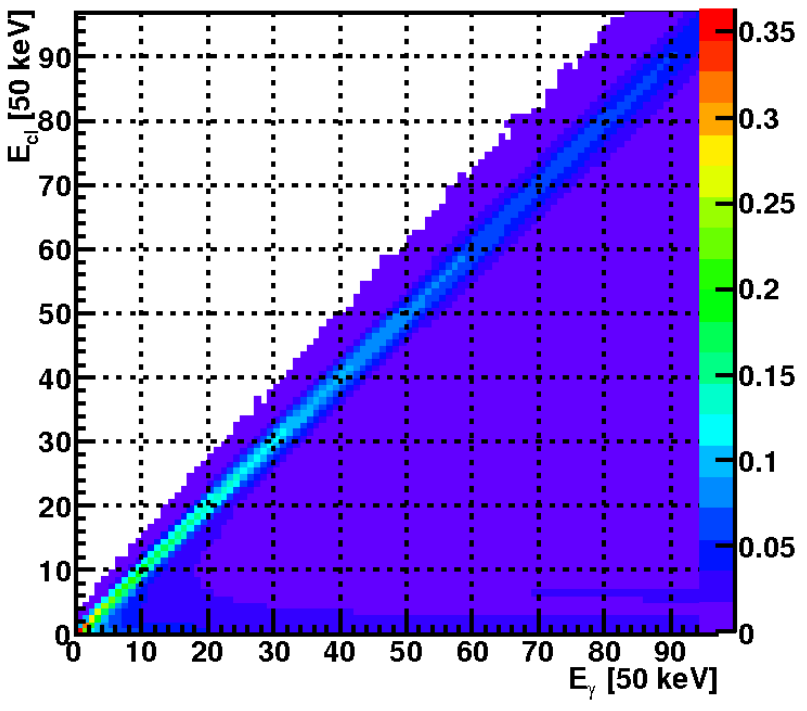

FIG. 10: E-response matrix of DANCE. X-axis labels are bin numbers. Each bin is $50 \mathrm{keV}$ wide.

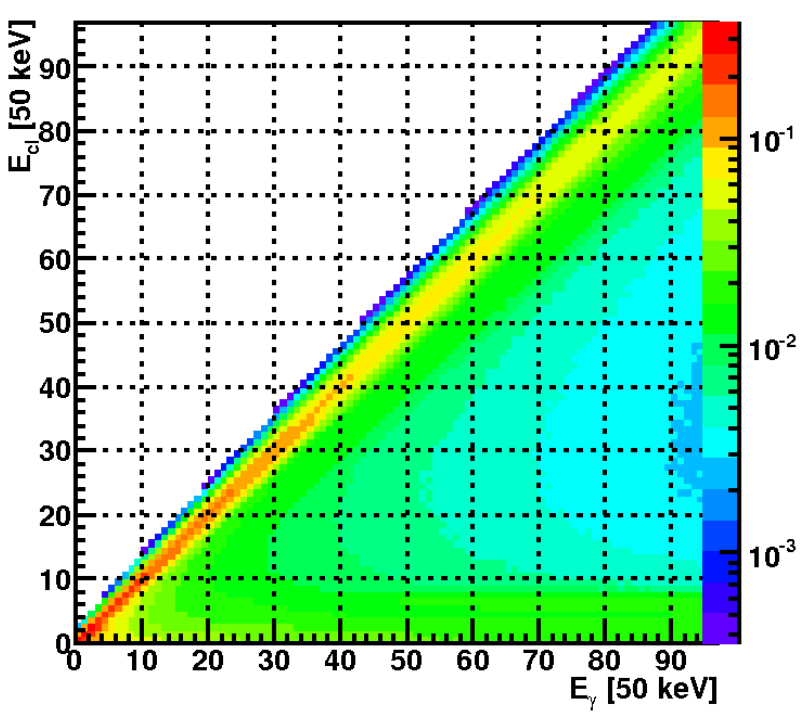

FIG. 11: E-response matrix of DANCE. Z-axis log view.

to make sure the entire range of interest covered. In many nuclei the neutron capture and neutron induced fission reactions produce $\gamma$-rays within $0.15-5 \mathrm{MeV}$. The GEANT4 model of DANCE was validated up to $3 \mathrm{MeV}$ using the calibration sources and the extrapolation is employed for the higher energy. The E-matrix can be used to reconstruct the true $\mathrm{E}_{\gamma}$ energy from the measured $\mathrm{E}_{c l}$ spectrum.

\section{Energy unfolding}

The DANCE energy response folds the true energy spectrum as follows:

$$
V\left(E_{c l}\right)=R(E) \cdot V\left(E_{\gamma}\right)
$$

where $V\left(E_{c l}\right)$ is the measured energy spectrum, $V\left(E_{\gamma}\right)$ is the true energy spectrum, and $R(E)$ is the E-response matrix. The energy unfolding is done the same way as the multiplicity unfolding. Eq. 4 is solved relative to the vector $V\left(E_{\gamma}\right)$ by factorizing the matrix $R(E)$ using the Singular Value Decomposition (SVD) method [5].

The energy unfolding has been made for the three calibration sources: ${ }^{60}$ Co (Fig. 12), ${ }^{88} \mathrm{Y}$ (Fig. 13), and ${ }^{22} \mathrm{Na}$ (Fig. 14). Each figure contain two spectra: the measured one in black and the unfolded one in red. 

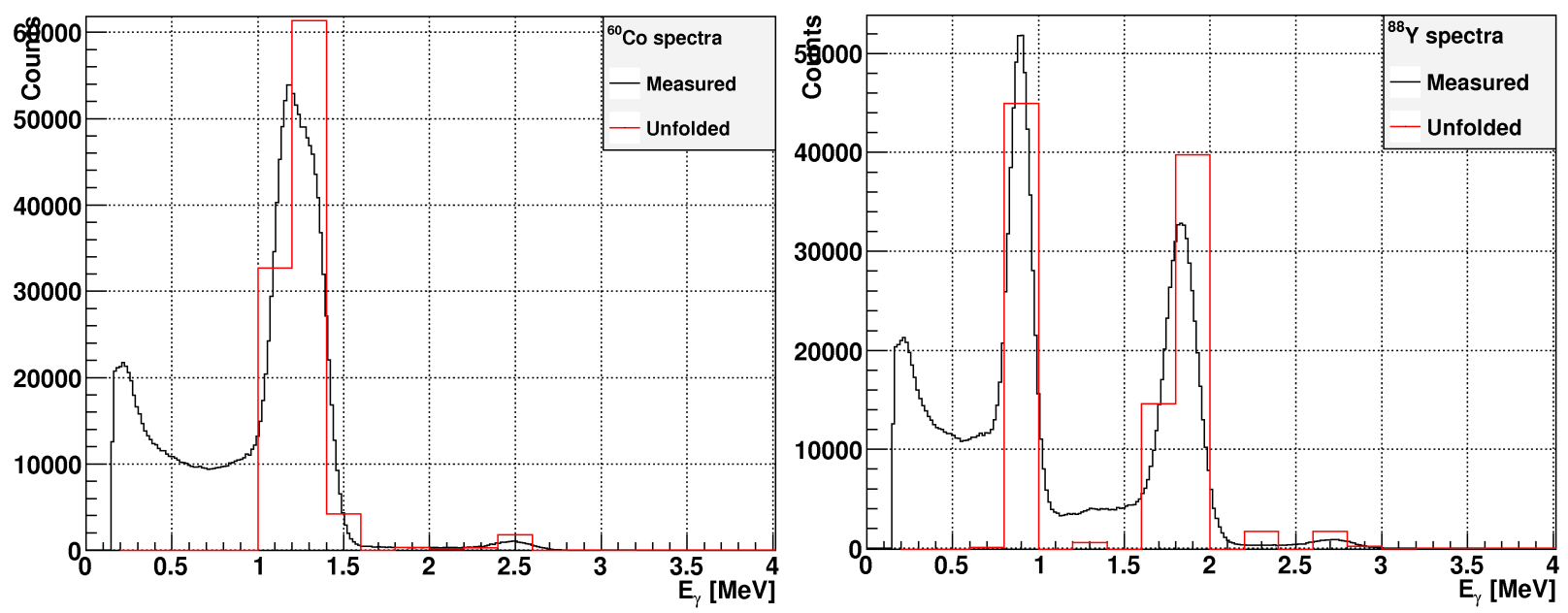

FIG. 12: Unfolding the true $\mathrm{E}_{\gamma}$ from the measured $\mathrm{E}_{c l}$ for ${ }^{60} \mathrm{Co}$.

FIG. 13: Unfolding the true $\mathrm{E}_{\gamma}$ from the measured $\mathrm{E}_{c l}$ for ${ }^{88} \mathrm{Y}$.

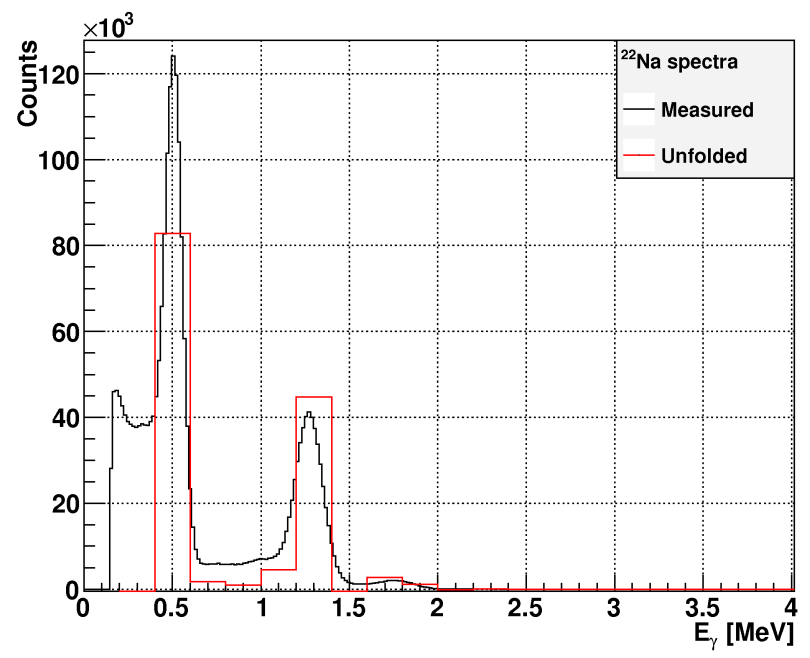

FIG. 14: Unfolding the true $\mathrm{E}_{\gamma}$ from the measured $\mathrm{E}_{c l}$ for ${ }^{22} \mathrm{Na}$.

The binning of the measured spectra is $16 \mathrm{keV}$ and $200 \mathrm{keV}$ for the unfolded spectra. The energy unfolding improves not only the energy resolution, but also the signal-to-noise ratio, that is, one can almost entirely eliminate compton background and get a clean peak of $\mathrm{E}_{\gamma}$. More importantly, the relative intensity ratio of the unfolded $\mathrm{E}_{\gamma}$ peaks is conserved for each spectrum. Note that the unfolding for the $100 \mathrm{keV}$ bin $\gamma$-ray spectrum also works well.

There is no doubt that the unfolding of the $\gamma$-ray energy and multiplicity distribution from the DANCE measurement is imperfect since approximations mentioned above are made for the detector response to measure these quantities. However, one still can obtain a 
reasonable solution for the true distribution under certain favorable conditions for unfolding. For instance, the modeling of the DANCE response to the $\gamma$-ray efficiency and multiplicity can be made reasonably well using the known $\gamma$-ray calibration sources. The nearly $\gamma$-ray energy independence of the detector response, discovered during the modeling stage, is the most important ingredient for the success of this proposed unfolding process. We are going to continue to explore various approaches to refine the unfolding method and achieve a better solution for the prompt $\gamma$-ray energy and multiplicity distribution in fission.

\section{DISCUSSION}

For CY2010 we have two scheduled experiments to measure the neutron-induced reactions on ${ }^{239} \mathrm{Pu}$ and ${ }^{241} \mathrm{Pu}$ using the DANCE array in conjunction with a new fission PPAC, designed and fabricated at LLNL. These provide an excellent opportunity to study the fission prompt $\gamma$-ray energy and multiplicity distribution in the Pu isotopes. In addition, we

are going to install a ${ }^{252} \mathrm{Cf}$ fission PPAC to study the prompt $\gamma$-ray energy and multiplicity distribution in spontaneous fission, which complements to our study for the neutron-induced fission. It certaintly will enhance our understanding of the $\gamma$-ray production in fission and benefit not only the modeling effort but also the nuclear power industry.

This work performed under the auspices of the US Department of Energy by Lawrence Livermore National Laboratory LLC under Contract DE-AC52-07NA27344 and by Los Alamos National Security LLC under contract DE-AC52-06NA25396.

[1] M. Heil, R. Reifarth, M.M. Fowler, R.C. Haight, F. Kappeler,R.S. Rundberg,E.H. Seabury, J.L. Ullmann, J.B. Wilhelmy, K. Wishhak.

$A 4 \pi \mathrm{BaF}_{2}$ detector for $(n, \gamma)$ cross-section measurements at a spallation neutron source. Nuclear Instruments and Methods in Physics Research A 459 (2001) 229-246.

[2] M. Jandel, T.A. Bredeweg, A. Couture, M.M. Fowler, E.M. Bond, M.B. Chadwick, R.R.C. Clement, E.-I. Esch, J.M. ODonnell, R. Reifarth, R.S. Rundberg, J.L. Ullmann, D.J. Vieira, J.B. Wilhelmy, J.M. Wouters, R.A. Macri, C.Y. Wu, J.A. Becker.

GEANT4 simulations of the DANCE array.

Nuclear Instruments and Methods in Physics Research Section B: Beam Interactions with Materials and Atoms, Volume 261, Issues 1-2, August 2007, Pages 1117-1121.

[3] A. Chyzh, C.Y. Wu, T. Bredeweg, A. Couture, M. Jandel, J. Ullmann, E. Norman. 
Fission prompt $\gamma$-ray multiplicity distribution measurements and simulations at DANCE. Lawrence Livermore National Laboratory, LLNL-TR-452298, Technical report, 2010.

[4] Glenn Samuel Brunson Jr.

Multiplicity and Correlation Energy of Gamma Rays Emitted in the Spontaneous Fission of Californium-252.

Los Alamos National Laboratory, LA-940B-T, Thesis.

http://www.osti.gov/bridge/purl.cover.jsp; jsessionid=5063EB75701F54FA5104139F225708D9? purl=/5187089-vSxswe/

[5] H. Golub Gene, W. Kahan.

"Calculating the singular values and pseudo-inverse of a matrix".

Journal of the Society for Industrial and Applied Mathematics: Series B, Numerical Analysis 2 (2): 205224, doi:10.1137/0702016.

http://www.jstor.org/stable/2949777 